\title{
Hedging and the competitive firm under correlated price and background risk
}

\author{
Kit Pong Wong
}

Received: 20 April 2012 / Accepted: 28 September 2012 / Published online: 10 October 2012 C The Author(s) 2012. This article is published with open access at Springerlink.com

\begin{abstract}
This paper examines the behavior of the competitive firm under correlated price and background risk when a futures market exists for hedging purposes. We show that imposing the background risk, be it additive or multiplicative, on the firm has no effect on the separation theorem. The full-hedging theorem, however, holds if the background risk is independent of the price risk. In the general case of the correlated price and background risk, we adopt the concept of expectation dependence to describe the bivariate dependence structure. When the background risk is additive, the firm finds it optimal to opt for an over-hedge or an under-hedge, depending on whether the price risk is positively or negatively expectation dependent on the background risk, respectively. When the background risk is multiplicative, both the concept of expectation dependence and the Arrow-Pratt measure of relative risk aversion are called for to determine the firm's optimal futures position.
\end{abstract}

Keywords Background risk $\cdot$ Expectation dependence $\cdot$ Hedging $\cdot$ Production

JEL Classification D21 D $81 \cdot$ G13

\section{Introduction}

Since the seminal work of Sandmo (1971), the theory of the competitive firm under price uncertainty has been the subject of considerable research in decision making

I would like to thank Paolo Ghirardato (the Editor-in-Chief), Frank Riedel (the Associate Editor), and an anonymous referee for their helpful comments and suggestions. The usual disclaimer applies.

K. P. Wong $(\bowtie)$

School of Economics and Finance, University of Hong Kong,

Pokfulam Road, Hong Kong, China

e-mail: kpwong@econ.hku.hk 
under uncertainty. One important strand of this literature examines the behavior of the firm when a futures market exists for hedging purposes, from which two celebrated results emanate (see, e.g., Adam-Müller 1997; Broll 1992; Broll and Zilcha 1992; Danthine 1978; Feder et al. 1980; Holthausen 1979; Wong 2003; to name just a few). First, the separation theorem states that the firm's production decision depends neither on the risk attitude of the firm, nor on the incidence of the price uncertainty. Second, the full-hedging theorem states that the firm fully hedges against its risk exposure to the price uncertainty should the futures market be unbiased. ${ }^{1}$

This paper contributes to the extant literature by incorporating additional sources of uncertainty that are aggregated into additive and/or multiplicative background risk with zero mean. Examples of additive background risk include the firm's initial wealth that is held in risky assets (Chavas 1985; Wong 1996), and the firm's fixed cost that is subject to shocks (Machnes 1993; Wong 1995). Examples of multiplicative background risk include revenue risk (Adam-Müller 1997; Broll and Wong 2012; Wong 2003), credit risk (Wong 1997), and inflation risk (Adam-Müller 2000; Battermann and Broll 2001). The background risk, be it additive or multiplicative, is not necessarily independent of the price risk. Given that the firm's production decision does not depend on the underlying uncertainty when a futures market exists for hedging purposes, it follows immediately that the separation theorem is robust to the introduction of the correlated background risk. The full-hedging theorem, however, holds if the background risk is independent of the price risk. Since the unbiased futures contracts cannot cross-hedge the independent background risk, it remains optimal to completely eliminate the risk exposure to the price uncertainty via a full-hedge (Adam-Müller 2000; Briys et al. 1993).

In the general case that the background risk is correlated with the price risk, we show that the concept of expectation dependence (Wright 1987) plays a pivotal role in determining the firm's optimal futures position. ${ }^{2}$ When the background risk is additive, the firm finds it optimal to opt for an over-hedge or an under-hedge, depending on whether the price risk is positively or negatively expectation dependent on the background risk, respectively. Wong (2012a) allows the background risk to be state dependent in that the magnitude of the background risk is gauged by a deterministic function of the realized state (Fei and Schlesinger 2008). In this more general setting, Wong (2012a) shows that prudence in the sense of Kimball $(1990,1993)$ is needed so as to make the firm's optimal futures position determinate.

When the background risk is multiplicative, we show that the concept of expectation dependence and the Arrow-Pratt measure of relative risk aversion jointly determine the firm's optimal futures position. Specifically, if the price risk is positively expectation dependent on the background risk, the firm finds it optimal to opt for an over-hedge or an under-hedge, depending on whether the firm's measure of relative risk aversion is everywhere greater or smaller than unity, respectively. On the other hand, if the price risk is negatively expectation dependent on the background risk, the firm finds

\footnotetext{
1 The full-hedging theorem is analogous to a well-known result in the insurance literature that a risk-averse individual fully insures at an actuarially fair price (Mossin 1968).

2 See Hong et al. (2011), Li (2011), and Wong (2012b, 2013) for other applications of expectation dependence.
} 
it optimal to opt for an over-hedge or an under-hedge, depending on whether the firm's measure of relative risk aversion is everywhere smaller or greater than unity, respectively. Adam-Müller (2000) uses a regression model to describe the price and background risk, which is a special case of expectation dependence. His results as such are generalized in this paper to much weaker dependence structure.

In a closely related paper, Adam-Müller and Nolte (2011) model multiplicative background risk in an asymmetric manner in that it is incorporated into either the spot price specification or the futures price specification, which is the right way to model basis risk. On the other hand, we introduce multiplicative background risk to affect both the spot and futures prices in a symmetric manner, which is more suitable to model credit risk or inflation risk. Because of the asymmetric treatment of the multiplicative background risk on the firm's payoff function, Adam-Müller and Nolte (2011) show that prudence in the sense of Kimball $(1990,1993)$ is called for to yield unambiguous futures positions.

The rest of this paper is organized as follows: Section 2 delineates the model of the competitive firm under correlated price and background risk. The firm has access to futures contracts to hedge against its risk exposure to the price uncertainty. Section 3 characterizes the firm's optimal production decision. Section 4 examines the firm's optimal hedging decision when the futures contracts are unbiased. Section 5 provides the conclusions.

\section{The model}

Consider the competitive firm under price uncertainty à la Sandmo (1971). There is one period with two dates, 0 and 1 . To begin, the firm produces a single commodity according to a deterministic cost function, $C(Q)$, where $Q \geq 0$ is the output level chosen by the firm at date 0 , and $C(Q)$ is compounded to date 1 . We assume that the cost function, $C(Q)$, satisfies that $C(0)=C^{\prime}(0)=0$, and that $C^{\prime}(Q)>0$ and $C^{\prime \prime}(Q)>0$ for all $Q>0 .^{3}$

At date 1 , the firm sells its entire output, $Q$, at the then prevailing per-unit price, $\tilde{P}$, that is not known ex ante. ${ }^{4}$ We denote $F(P)$ as the marginal cumulative distribution function (CDF) of $\tilde{P}$ over support $[\underline{P}, \bar{P}]$, where $0<\underline{P}<\bar{P}$. The firm can trade infinitely divisible futures contracts at date 0 to hedge against its risk exposure to $\tilde{P}$. Each futures contract calls for delivery of one unit of the commodity at date 1 at the predetermined futures price, $P^{f} \in(\underline{P}, \bar{P})$. Let $X$ be the number of the futures contracts sold (purchased if negative) by the firm at date 0 . The futures position, $X$, is said to be an under-hedge, a full-hedge, or an over-hedge, depending on whether $X$ is smaller than, equal to, or greater than the output level, $Q$, respectively.

Besides the price uncertainty, the firm faces other sources of uncertainty that are aggregated into additive and/or multiplicative background risk, $\tilde{Z}$, with a mean set equal to zero. We denote $G(Z)$ as the marginal $\mathrm{CDF}$ of $\tilde{Z}$ over support $[\underline{Z}, \bar{Z}]$, where

\footnotetext{
${ }^{3}$ The strict convexity of $C(Q)$ is driven by the firm's production technology that exhibits decreasing returns to scale.

4 Throughout the paper, random variables have a tilde $(\sim)$, while their realizations do not.
} 
$\underline{Z}<0<\bar{Z}$. To allow for possible correlation between $\tilde{P}$ and $\tilde{Z}$, we denote $H(P, Z)$ as their joint $\mathrm{CDF}$ over support $[\underline{P}, \bar{P}] \times[\underline{Z}, \bar{Z}]$. The two random variables are independent if, and only if, $H(P, Z)=F(P) G(Z)$ for all $(P, Z) \in[\underline{P}, \bar{P}] \times[\underline{Z}, \bar{Z}]$. Unlike the price risk, $\tilde{P}$, the background risk, $\tilde{Z}$, is neither hedgeable nor insurable. The firm's random profit at date 1 is, therefore, given by

$$
\tilde{\Pi}=(1+\beta \tilde{Z})\left[\tilde{P} Q+\left(P^{f}-\tilde{P}\right) X-C(Q)+(1-\beta) \tilde{Z}\right],
$$

where $\beta \in[0,1]$ is a constant such that $-\beta \underline{Z}<1$. If $\beta=0$ or 1 , the background risk becomes purely additive or purely multiplicative, respectively.

The firm possesses a von Neumann-Morgenstern utility function, $U(\Pi)$, defined over its profit, $\Pi$, at date 1 . The firm is risk averse so that $U^{\prime}(\Pi)>0$ and $U^{\prime \prime}(\Pi)<0$ for all $\Pi>0$. The ex-ante decision problem faced by the firm at date 0 is to choose an output level, $Q \geq 0$, and a futures position, $X$, so as to maximize the expected utility of its profit at date 1 :

$$
\max _{Q \geq 0, X} \mathrm{E}[U(\tilde{\Pi})]
$$

where $\mathrm{E}(\cdot)$ is the expectation operator with respect to the joint $\mathrm{CDF}, H(P, Z)$, and $\tilde{\Pi}$ is given by Eq. (1).

The first-order conditions for program (2) are given by

$$
\mathrm{E}\left\{U^{\prime}\left(\tilde{\Pi}^{*}\right)(1+\beta \tilde{Z})\left[\tilde{P}-C^{\prime}\left(Q^{*}\right)\right]\right\}=0,
$$

and

$$
\mathrm{E}\left[U^{\prime}\left(\tilde{\Pi}^{*}\right)(1+\beta \tilde{Z})\left(P^{f}-\tilde{P}\right)\right]=0
$$

where an asterisk $\left(^{*}\right)$ indicates an optimal level. The second-order conditions for program (2) are satisfied given that $U^{\prime \prime}(\Pi)<0$ and $C^{\prime \prime}(Q)>0$.

\section{Optimal production decision}

In this section, we examine the firm's optimal production decision. Adding Eqs. (3)-(4) yields

$$
\mathrm{E}\left[U^{\prime}\left(\tilde{\Pi}^{*}\right)(1+\beta \tilde{Z})\right]\left[P^{f}-C^{\prime}\left(Q^{*}\right)\right]=0
$$

Given that $U^{\prime}(\Pi)>0$, Eq. (5) reduces to $C^{\prime}\left(Q^{*}\right)=P^{f}$, thereby invoking our first proposition. All proofs of propositions are relegated to the Appendix.

Proposition 1 If the competitive firm that faces the correlated price and background risk can trade the futures contracts for hedging purposes, the firm's optimal output level, $Q^{*}$, is the one at which the marginal cost of production, $C^{\prime}\left(Q^{*}\right)$, is equated to the predetermined futures price, $P^{f}$. 
The intuition for Proposition 1 is as follows. By producing one more unit of the commodity, the firm receives the marginal revenue, $(1+\beta \tilde{Z}) \tilde{P}$, which is stochastic. The firm can sell this additional unit forward via trading one futures contract to lock in the marginal revenue at $(1+\beta \tilde{Z}) P^{f}$, which remains stochastic due to the multiplicative background risk. At the optimum, the firm equates the marginal revenue, $(1+\beta \tilde{Z}) P^{f}$, to the marginal cost, $(1+\beta \tilde{Z}) C^{\prime}\left(Q^{*}\right)$. This then gives rise to the usual optimality condition, $C^{\prime}\left(Q^{*}\right)=P^{f}$, that determines the optimal output level, $Q^{*}$.

An immediate implication of Proposition 1 is that the firm's optimal production decision depends neither on the utility function, nor on the multiple sources of uncertainty. Proposition 1 as such extends the separation theorem to the case in which there is background risk that is correlated with the price uncertainty.

\section{Optimal hedging decision}

In this section, we characterize the firm's optimal hedging decision. To focus on the firm's pure hedging motive, we assume hereafter that the futures contracts are unbiased in that the futures price, $P^{f}$, is set equal to the unconditional expected value of the random per-unit price, $\tilde{P}$, that is,

$$
P^{f}=\int_{\underline{P}}^{\bar{P}} P \mathrm{~d} F(P) .
$$

Given Eq. (6), we can write Eq. (4) as ${ }^{5}$

$$
\operatorname{Cov}\left[U^{\prime}\left(\tilde{\Pi}^{*}\right)(1+\beta \tilde{Z}), \tilde{P}\right]=0,
$$

where $\operatorname{Cov}(\cdot, \cdot)$ is the covariance operator with respect to the joint $\operatorname{CDF}, H(P, Z)$.

Let $R(\Pi)=-\Pi U^{\prime \prime}(\Pi) / U^{\prime}(\Pi)$ for all $\Pi>0$ be the Arrow-Pratt measure of relative risk aversion. Denote $\Pi^{*}(Z)=(1+\beta Z)\left[P^{f} Q^{*}-C\left(Q^{*}\right)+(1-\beta) Z\right]$. We derive necessary and sufficient conditions that guarantee the optimality of an underhedge $\left(X^{*}<Q^{*}\right)$, a full-hedge $\left(X^{*}=Q^{*}\right)$, and an over-hedge $\left(X^{*}>Q^{*}\right)$ in the following proposition.

Proposition 2 Given that the competitive firm can trade the unbiased futures contracts for hedging purposes, the firm's optimal futures position, $X^{*}$, is smaller than, equal to, or greater than the optimal output level, $Q^{*}$, if, and only if,

$$
\begin{aligned}
& \operatorname{Cov}\left\{U^{\prime}\left[\Pi^{*}(\tilde{Z})\right](1+\beta \tilde{Z}), \tilde{P}\right\}=\int_{\underline{P}}^{\bar{P}} \int_{Z}^{\bar{Z}}[H(P, Z)-F(P) G(Z)] \\
& \times\left\{\beta\left\{1-R\left[\Pi^{*}(Z)\right]\right\} U^{\prime}\left[\Pi^{*}(Z)\right]+(1-\beta)(1+\beta Z)^{2} U^{\prime \prime}\left[\Pi^{*}(Z)\right]\right\} \mathrm{d} P \mathrm{~d} Z
\end{aligned}
$$

is positive, zero, or negative, respectively.

\footnotetext{
${ }^{5}$ For any two random variables, $\tilde{X}$ and $\tilde{Y}$, we have $\operatorname{Cov}(\tilde{X}, \tilde{Y})=\mathrm{E}(\tilde{X} \tilde{Y})-\mathrm{E}(\tilde{X}) \mathrm{E}(\tilde{Y})$.
} 
The right-hand side of Eq. (8), albeit complicated in its general form, can be simplified to provide intuitive hedging rules to the firm. The following proposition stipulates sufficient conditions under which a full-hedge $\left(X^{*}=Q^{*}\right)$ is optimal.

Proposition 3 Given that the competitive firm can trade the unbiased futures contracts for hedging purposes, the firm optimally opts for a full-hedge, that is, $X^{*}=Q^{*}$, if the random per-unit price, $\tilde{P}$, and the background risk, $\tilde{Z}$, are independent, or if the firm has a logarithmic utility function, that is, $U(\Pi)=\ln \Pi$, and the background risk is purely multiplicative, that is, $\beta=0$.

The intuition for Proposition 3 is as follows. Equation(7) implies that the optimal futures position, $X^{*}$, is the one that makes the multiple of the firm's marginal utility, $U^{\prime}\left(\tilde{\Pi}^{*}\right)$, and the multiplicative background risk, $1+\beta \tilde{Z}$, invariant to the random perunit price, $\tilde{P}$. By adopting a full-hedge, that is, $X^{*}=Q^{*}$, the firm's profit at date 1 is given by $(1+\beta \tilde{Z})\left[P^{f} Q^{*}-C\left(Q^{*}\right)+(1-\beta) \tilde{Z}\right]$, which depends only on the background risk, $\tilde{Z}$. If $\tilde{P}$ and $\tilde{Z}$ are independent, there is no residual hedgeable risk that can be eliminated by trading further the futures contracts, thereby rendering the optimality of a full-hedge. On the other hand, if the firm has a logarithmic utility function so that $U^{\prime}(\Pi)=1 / \Pi$, and the background risk is purely multiplicative so that $\beta=1$, a full-hedge implies that the multiple, $U^{\prime}\left(\tilde{\Pi}^{*}\right)(1+\beta \tilde{Z})$, becomes $1 /\left[P^{f} Q^{*}-C\left(Q^{*}\right)\right]$, which is non-stochastic. Hence, a full-hedge is indeed optimal in this case irrespective of how $\tilde{P}$ and $\tilde{Z}$ are correlated.

To derive sufficient conditions under which an under-hedge $\left(X^{*}<Q^{*}\right)$ or an overhedge $\left(X^{*}>Q^{*}\right)$ is optimal, we need to consider some tractable dependence structure on $\tilde{P}$ and $\tilde{Z}$. To this end, we define the CDF of $\tilde{P}$ conditional on the event that $\tilde{Z} \leq Z$ as

$$
F(P \mid \tilde{Z} \leq Z)=\frac{H(P, Z)}{G(Z)}
$$

over support $[\underline{P}, \bar{P}]$ for all $Z \in[\underline{Z}, \bar{Z}]$. Let $\mathrm{E}(\tilde{P} \mid \tilde{Z} \leq Z)$ be the expected value of $\tilde{P}$ with respect to the conditional $\mathrm{CDF}, F(P \mid \tilde{Z} \leq \bar{Z})$. The following bivariate dependence structure, known as expectation dependence, is due to Wright (1987).

Definition 1 The random per-unit price, $\tilde{P}$, is said to be positively (negatively) expectation dependent on the background risk, $\tilde{Z}$, if

$$
\mathrm{ED}(\tilde{P} \mid Z)=\mathrm{E}(\tilde{P})-\mathrm{E}(\tilde{P} \mid \tilde{Z} \leq Z) \geq(\leq) 0
$$

for all $Z \in[\underline{Z}, \bar{Z}]$, where the inequality is strict for some non-degenerate intervals.

Equation(10) implies that the expected value of the random per-unit price, $\tilde{P}$ is revised upward (downward) whenever one discovers that the background risk, $\tilde{Z}$, is small, in the precise sense that one is given the truncation, $\tilde{Z} \leq Z$. To see further how Definition 1 defines dependence, we write Eq. (10) as 


$$
\begin{aligned}
\mathrm{ED}(\tilde{P} \mid Z) & =\int_{\underline{P}}^{\bar{P}} P \mathrm{~d} F(P)-\int_{P}^{\bar{P}} \mathrm{~d} F(P \mid \tilde{Z} \leq Z) \\
& =\int_{\underline{P}}^{\bar{P}}[F(P \mid \tilde{Z} \leq Z)-F(P)] \mathrm{d} P,
\end{aligned}
$$

where the second equality follows from integration by parts. It is evident from Eq. (11) that $\operatorname{ED}(\tilde{P} \mid Z) \geq(\leq) 0$ if $F(P \mid \tilde{Z} \leq Z) \geq(\leq) F(P)$ for all $P \in[\underline{P}, \bar{P}]$, that is, if $F(P)$ dominates (is dominated by) $F(P \mid \bar{Z} \leq Z)$ in the sense of first-order stochastic dominance. Hence, positive (negative) expectation dependence is implied by the fact that small background risk increases (decreases) the riskiness of the random per-unit price in the sense of first-order stochastic dominance, which can be verified empirically using tests of stochastic dominance.

According to Lehmann (1966), we can write $\operatorname{Cov}(\tilde{P}, \tilde{Z})$ in terms of the $\operatorname{CDFs}$, $F(P), G(Z)$, and $H(P, Z)$ :

$$
\begin{aligned}
\operatorname{Cov}(\tilde{P}, \tilde{Z}) & =\int_{\underline{P}}^{\bar{P}} \int_{\bar{Z}}^{\bar{Z}}[H(P, Z)-F(P) G(Z)] \mathrm{d} P \mathrm{~d} Z \\
& =\int_{\underline{Z}}^{\bar{Z}}\left\{\int_{\underline{P}}^{\bar{P}}[F(P \mid \tilde{Z} \leq Z)-F(P)] \mathrm{d} P\right\} G(Z) \mathrm{d} Z \\
& =\int_{\bar{Z}} \operatorname{ED}(\tilde{P} \mid Z) G(Z) \mathrm{d} Z,
\end{aligned}
$$

where the second equality follows from Eq. (9), and the last equality follows from Eq. (11). From Definition 1 and Eq. (12), we have $\operatorname{Cov}(\tilde{P}, \tilde{Z})>(<) 0$ if $\tilde{P}$ is positively (negatively) expectation dependent on $\tilde{Z} .^{6}$

Equipped with the concept of expectation dependence, we derive the firm's optimal futures position, $X^{*}$, in the following proposition when the background risk is purely additive, that is, $\beta=0$.

Proposition 4 Given that the competitive firm can trade the unbiased futures contracts for hedging purposes and that the background risk, $\tilde{Z}$, is purely additive, that is, $\beta=0$, the firm optimally opts for an over-hedge (under-hedge), that is, $X^{*}>(<) Q^{*}$, if the random per-unit price, $\tilde{P}$, is positively (negatively) expectation dependent on $\tilde{Z}$.

\footnotetext{
${ }^{6} \mathrm{Li}$ (2011) and Wright (1987) show that two random variables that are positively (negatively) correlated need not be positively (negatively) expectation dependent. Hence, positive (negative) expectation dependence is stronger than positive (negative) correlation.
} 
The intuition for Proposition 4 is as follows. Equation(7) with $\beta=0$ implies that the optimal futures position, $X^{*}$, is the one that makes the firm's marginal utility, $U^{\prime}\left(\tilde{\Pi}^{*}\right)$, invariant to the random per-unit price, $\tilde{P}$. If $\tilde{P}$ and $\tilde{Z}$ are positively (negatively) correlated in the sense of expectation dependence, a full-hedge implies that $\operatorname{Cov}\left\{U^{\prime}\left[P^{f} Q^{*}-C\left(Q^{*}\right)+\tilde{Z}\right], \tilde{P}\right\}<(>) 0$. An over-hedge (under-hedge) reduces the firm's profit at date 1 as $P$ increases (decreases), which is more likely when $Z$ is higher. Given risk aversion, such a futures position is more effective in reducing the variability of the firm's marginal utility, thereby rendering the optimality of an over-hedge (under-hedge) if $\tilde{P}$ is positively (negatively) expectation dependent on $\tilde{Z}$.

We now turn to the case that the background risk is purely multiplicative, that is, $\beta=1$. We derive sufficient conditions under which an under-hedge $\left(X^{*}<Q^{*}\right)$ or an over-hedge $\left(X^{*}>Q^{*}\right)$ is optimal.

Proposition 5 Given that the competitive firm can trade the unbiased futures contracts for hedging purposes and that the background risk, $\tilde{Z}$, is purely multiplicative, that is, $\beta=1$, the firm optimally opts for an over-hedge, that is, $X^{*}>Q^{*}$, if the random per-unit price, $\tilde{P}$, is positively (negatively) expectation dependent on $\tilde{Z}$ and the ArrowPratt measure of relative risk aversion, $R(\Pi)$, is everywhere no less (no greater) than unity. An under-hedge, that is, $X^{*}<Q^{*}$, is optimal if $\tilde{P}$ is positively (negatively) expectation dependent on $\tilde{Z}$ and $R(\Pi) \leq(\geq) 1$ for all $\Pi>0$.

The intuition for Proposition 5 is as follows. Equation (7) with $\beta=1$ implies that the optimal futures position, $X^{*}$, is the one that makes the multiple of the firm's marginal utility, $U^{\prime}\left(\tilde{\Pi}^{*}\right)$, and the multiplicative background risk, $1+\tilde{Z}$, invariant to the random per-unit price, $\tilde{P}$. Consider first the case that $\tilde{P}$ is positively expectation dependent on $\tilde{Z}$. In this case, the firm has incentives to opt for an over-hedge so as to cross-hedge against $\tilde{Z}$. We refer to this as the over-hedging effect. On the other hand, an underhedge increases (reduces) the firm's profit at date 1 as $P$ increases (decreases), which is more likely when $Z$ is higher (lower). Given risk aversion, such a futures position is more effective in reducing the variability of $U^{\prime}\left(\tilde{\Pi}^{*}\right)(1+\tilde{Z})$. We refer to this as the under-hedging effect. Since the elasticity of the firm's marginal utility is gauged by the Arrow-Pratt measure of relative risk aversion, $R(\Pi)=-\Pi U^{\prime \prime}(\Pi) / U^{\prime}(\Pi)$, the firm's marginal utility is (not) sensitive to changes in profit if $R(\Pi)$ is large (small). In this case, the over-hedging effect dominates (is dominated by) the under-hedging effect in determining the firm's optimal futures position, thereby rendering the optimality of an over-hedge (under-hedge) when $\tilde{P}$ is positively expectation dependent on $\tilde{Z}$. Similar intuition applies to the optimality of an over-hedge (under-hedge) if $\tilde{P}$ is negatively expectation dependent on $\tilde{Z}$ and $R(\Pi)$ is small (large).

Finally, we consider the general case that $\beta \in(0,1)$. The firm's optimal futures position, $X^{*}$, is characterized in the following proposition.

Proposition 6 Given that the competitive firm can trade the unbiased futures contracts for hedging purposes and that the background risk, $\tilde{Z}$, is both additive and multiplicative, that is, $0<\beta<1$, the firm optimally opts for an over-hedge (underhedge), that is, $X^{*}>(<) Q_{\tilde{Z}}^{*}$, if the random per-unit price, $\tilde{P}$, is positively (negatively) expectation dependent on $\tilde{Z}$ and the Arrow-Pratt measure of relative risk aversion, $R(\Pi)$, is everywhere no less than unity. 
Proposition 6 follows immediately from combining the sufficient conditions derived in Propositions 4 and 5 to determine the firm's optimal futures position, $X^{*}$.

\section{Conclusion}

This paper examines the behavior of the competitive firm under price uncertainty $\grave{a}$ la Sandmo (1971) when background risk is present and correlated with the price risk. We show that the separation theorem is robust to the introduction of the correlated background risk, while the full-hedging theorem is not. Specifically, the full-hedging theorem holds if the background risk is independent of the price risk. In the general case when these two sources of uncertainty are correlated, we show that the concept of expectation dependence (Wright 1987) plays a pivotal role in shaping the firm's optimal hedging decision. When the background risk is additive, the firm finds it optimal to opt for an over-hedge or an under-hedge, depending on whether the price risk is positively or negatively expectation dependent on the background risk, respectively. When the background risk is multiplicative, we show that the concept of expectation dependence and the Arrow-Pratt measure of relative risk aversion jointly determine the firm's optimal futures position. Given that multiple sources of uncertainty are a fact of life, there are many applications of expectation dependence that are worth exploring. We leave these for future research.

Open Access This article is distributed under the terms of the Creative Commons Attribution License which permits any use, distribution, and reproduction in any medium, provided the original author(s) and the source are credited.

\section{Appendix}

Proof of Proposition 1 See the text.

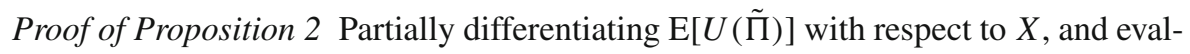
uating the resulting derivative at $Q=Q^{*}$ and $X=Q^{*}$ yields

$$
\left.\frac{\partial E[U(\tilde{\Pi})]}{\partial X}\right|_{Q=Q^{*}, X=Q^{*}}=-\operatorname{Cov}\left\{U^{\prime}\left[\Pi^{*}(\tilde{Z})\right](1+\beta \tilde{Z}), \tilde{P}\right\}
$$

where we have used Eq. (6), and $\Pi^{*}(\tilde{Z})=(1+\beta \tilde{Z})\left[P^{f} Q^{*}-C\left(Q^{*}\right)+(1-\beta) \tilde{Z}\right]$. It follows from Eq. (7) and the second-order conditions for program (2) that $X^{*}$ is less than, equal to, or greater than $Q^{*}$ if, and only if, the right-hand side of Eq. (13) is negative, zero, or positive, respectively. As shown by Cuadras $(2002), \operatorname{Cov}[A(\tilde{P}), B(\tilde{Z})]$ can be written in terms of the CDFs, $F(P), G(Z)$, and $H(P, Z)$ :

$$
\operatorname{Cov}[A(\tilde{P}), B(\tilde{Z})]=\int_{\underline{P}}^{\bar{P}} \int_{\underline{Z}}^{\bar{Z}}[H(P, Z)-F(P) G(Z)] \mathrm{d} A(P) \mathrm{d} B(Z),
$$


for all functions, $A(\cdot)$ and $B(\cdot)$. Equation (8) follows from Eq. (14) with $A(\tilde{P})=\tilde{P}$ and $B(\tilde{Z})=U^{\prime}\left[\Pi^{*}(\tilde{Z})\right](1+\beta \tilde{Z})$. The desired results immediately follow.

Proof of Proposition 3 If $\tilde{P}$ and $\tilde{Z}$ are independent, we have $H(P, Z)=F(P) G(Z)$ for all $(P, Z) \in[\underline{P}, \bar{P}] \times[\underline{Z}, \bar{Z}]$ so that expression (8) vanishes. On the other hand, if $U(\Pi)=\ln \Pi$, we have $R(\Pi) \equiv 1$ for all $\Pi>0$. It then follows from $\beta=1$ that expression (8) vanishes. In either case, Proposition 2 implies that $X^{*}=Q^{*}$.

Proof of Proposition 4 Since $\beta=0$, the right-hand side of Eq. (8) becomes

$$
\begin{aligned}
& \int_{\underline{Z}}^{\bar{Z}}\left\{\int_{\underline{P}}^{\bar{P}}[F(P \mid \tilde{Z} \leq Z)-F(P)] \mathrm{d} P\right\} U^{\prime \prime}\left[P^{f} Q^{*}-C\left(Q^{*}\right)+Z\right] G(Z) \mathrm{d} Z \\
& =\int_{\underline{Z}} \operatorname{ED}(\tilde{P} \mid Z) U^{\prime \prime}\left[P^{f} Q^{*}-C\left(Q^{*}\right)+Z\right] G(Z) \mathrm{d} Z,
\end{aligned}
$$

where the equality follows from Eq. (11). Given that $U^{\prime \prime}(\Pi)<0$, the right-hand side of Eq. (15) is negative (positive) if $\tilde{P}$ is positively (negatively) expectation dependent on $\tilde{Z}$, thereby implying that $X^{*}>(<) Q^{*}$ from Proposition 2 .

Proof of Proposition 5 Since $\beta=1$, the right-hand side of Eq. (8) becomes

$$
\begin{aligned}
& \int_{\underline{Z}}^{\bar{Z}}\left\{\int_{\underline{P}}^{\bar{P}}[F(P \mid \tilde{Z} \leq Z)-F(P)] \mathrm{d} P\right\} U^{\prime}\left\{(1+Z)\left[P^{f} Q^{*}-C\left(Q^{*}\right)\right]\right\} \\
& \quad \times\left\{1-R\left\{(1+Z)\left[P^{f} Q^{*}-C\left(Q^{*}\right)\right]\right\}\right\} G(Z) \mathrm{d} Z \\
& =\int_{\underline{Z}} \operatorname{ED}(\tilde{P} \mid Z) U^{\prime}\left\{(1+Z)\left[P^{f} Q^{*}-C\left(Q^{*}\right)\right]\right\} \\
& \quad \times\left\{1-R\left\{(1+Z)\left[P^{f} Q^{*}-C\left(Q^{*}\right)\right]\right\}\right\} G(Z) \mathrm{d} Z,
\end{aligned}
$$

where the second equality follows from Eq. (11). If $R(\Pi) \geq(\leq) 1$ for all $\Pi>$ 0 , the right-hand side of Eq. (16) has the opposite sign to (the same sign as) that of $\operatorname{ED}(\tilde{P} \mid Z)$. It follows from Proposition 2 that $X^{*}>(<) Q^{*}$ if $\tilde{P}$ is positively (negatively) expectation dependent on $\tilde{Z}$ and $R(\Pi) \geq 1$ for all $\Pi>0$, and that $X^{*}<(>) Q^{*}$ if $\tilde{P}$ is positively (negatively) expectation dependent on $\tilde{Z}$ and $R(\Pi) \leq 1$ for all $\Pi>0$. 
Proof of Proposition 6 We can write the right-hand side of Eq. (8) as

$$
\begin{aligned}
& \int_{\underline{Z}}^{\bar{Z}}\left\{\int_{\underline{P}}^{\bar{P}}[F(P \mid \tilde{Z} \leq Z)-F(P)] \mathrm{d} P\right\} \\
& \quad \times\left\{\beta U^{\prime}\left[\Pi^{*}(Z)\right]\left\{1-R\left[\Pi^{*}(Z)\right]\right\}+(1-\beta)(1+\beta Z)^{2} U^{\prime \prime}\left[\Pi^{*}(Z)\right]\right\} G(Z) \mathrm{d} Z \\
& =\int_{\bar{Z}} \operatorname{ED}(\tilde{P} \mid Z) \\
& \quad \times\left\{\beta U^{\prime}\left[\Pi^{*}(Z)\right]\left\{1-R\left[\Pi^{*}(Z)\right]\right\}+(1-\beta)(1+\beta Z)^{2} U^{\prime \prime}\left[\Pi^{*}(Z)\right]\right\} G(Z) \mathrm{d} Z,
\end{aligned}
$$

where the second equality follows from Eq. (11). If $R(\Pi) \geq 1$ for all $\Pi>0$, the right-hand side of Eq. (17) has the opposite sign to that of $\operatorname{ED}(\tilde{P} \mid Z)$. It follows from Proposition 2 that $X^{*}>(<) Q^{*}$ if $\tilde{P}$ is positively (negatively) expectation dependent on $\tilde{Z}$ and $R(\Pi) \geq 1$ for all $\Pi>0$.

\section{References}

Adam-Müller, A.F.A.: Export and hedging decisions under revenue and exchange rate risk: a note. Eur. Econ. Rev. 41, 1421-1426 (1997)

Adam-Müller, A.F.A.: Hedging price risk when real wealth matters. J. Int. Money Finance 19, 549-560 (2000)

Adam-Müller, A.F.A., Nolte, I.: Cross hedging under multiplicative basis risk. J. Bank. Finance 35, 29562964 (2011)

Battermann, H.L., Broll, U.: Inflation risk, hedging, and exports. Rev. Dev. Econ. 5, 355-362 (2001)

Briys, E., Crouhy, M., Schlesinger, H.: Optimal hedging in a futures market with background noise and basis risk. Eur. Econ. Rev. 37, 949-960 (1993)

Broll, U.: The effect of forward markets on multinational firms. Bull. Econ. Res. 44, 233-240 (1992)

Broll, U., Wong, K.P.: The firm under uncertainty: real and financial decisions. Decis. Econ. Finance 35, (2012, in press)

Broll, U., Zilcha, I.: Exchange rate uncertainty, futures markets and the multinational firm. Eur. Econ. Rev. 36, 815-826 (1992)

Chavas, J.-P.: On the theory of the competitive firm under uncertainty when initial wealth is random. S. Econ. J. 51, 818-827 (1985)

Cuadras, C.M.: On the covariance between functions. J, Multivar. Anal. 81, 19-27 (2002)

Danthine, J.-P.: Information, futures prices, and stabilizing speculation. J. Econ. Theory 17, 79-98 (1978)

Feder, G., Just, R.E., Schmitz, A.: Futures markets and the theory of the firm under price uncertainty. Q. J. Econ. 94, 317-328 (1980)

Fei, W., Schlesinger, H.: Precautionary insurance demand with state-dependent background risk. J. Risk Insur. 75, 1-16 (2008)

Holthausen, D.M.: Hedging and the competitive firm under price uncertainty. Am. Econ. Rev. 69, 989-995 (1979)

Hong, S.K., Lew, K.O., MacMinn, R., Brockett, P.: Mossin's Theorem given random initial wealth. J. Risk Insur. 78, 309-324 (2011)

Kimball, M.S.: Precautionary saving in the small and in the large. Econometrica 58, 53-73 (1990)

Kimball, M.S.: Standard risk aversion. Econometrica 61, 589-611 (1993) 
Lehmann, E.L.: Some concepts of dependence. Ann. Math. Stat. 37, 1137-1153 (1966)

$\mathrm{Li}, \mathrm{J}$. : The demand for a risky asset in the presence of a background risk. J. Econ. Theory 146, 372-391 (2011)

Machnes, Y.: Further results on comparative statics under uncertainty. Eur. J. Political Econ. 9, 141-146 (1993)

Mossin, J.: Aspects of rational insurance purchasing. J. Political Econ. 76, 553-568 (1968)

Sandmo, A.: On the theory of the competitive firm under price uncertainty. Am. Econ. Rev. 61, 65-73 (1971)

Wong, K.P.: Further results on comparative statics under uncertainty: a comment on Machnes. Eur. J. Political Econ. 11, 761-768 (1995)

Wong, K.P.: Background risk and the theory of the competitive firm under uncertainty. Bull. Econ. Res. 48, 241-251 (1996)

Wong, K.P.: On the determinants of bank interest margins under credit and interest rate risks. J. Bank. Finance 21, 251-271 (1997)

Wong, K.P.: Currency hedging with options and futures. Eur. Econ. Rev. 47, 833-839 (2003)

Wong, K.P.: Production and futures hedging with state-dependent background risk. Int. Rev. Econ. Finance 24, 177-184 (2012a)

Wong, K.P.: Production and hedging under state-dependent preferences. J. Futur. Mark. 32, 945-963 (2012b)

Wong, K. P.: Cross hedging with currency forward contracts. J. Futur. Mark. 33 (2013)

Wright, R.: Expectation dependence of random variables, with an application in portfolio theory. Theory Decis. 22, 111-124 (1987) 